
allemande

50-2 | 2018

Humanités environnementales - Quoi de neuf du côté des méthodes?

\title{
L'exposition artisanale : représentation et « mise en scène » de l'économie « nationale » en Transylvanie, 1868-1914
}

\section{Stéphanie Danneberg}

\section{(2) OpenEdition}

\section{Journals}

Édition électronique

URL : https://journals.openedition.org/allemagne/994

DOI : 10.4000/allemagne.994

ISSN : 2605-7913

Éditeur

Société d'études allemandes

Édition imprimée

Date de publication : 30 décembre 2018

Pagination : 441-456

ISSN : 0035-0974

Référence électronique

Stéphanie Danneberg, «L'exposition artisanale : représentation et « mise en scène » de l'économie «nationale » en Transylvanie, 1868-1914 », Revue d'Allemagne et des pays de langue allemande [En ligne], 50-2 | 2018, mis en ligne le 30 décembre 2019, consulté le 24 mai 2021. URL : http:// journals.openedition.org/allemagne/994 ; DOI : https://doi.org/10.4000/allemagne.994 


\section{L'exposition artisanale: représentation et «mise en scène» de l'économie «nationale» en Transylvanie, 1868-1914 ${ }^{(1)}$}

- Stéphanie Danneberg*

"C'est une belle exposition, pas seulement d'un grand intérêt pour les Saxons mais pour tous les citadins. Elle est également très instructive pour nos artisans et pour le peuple roumain ${ }^{(2)}$.

Si les associations transylvaines d'artisans roumains (Gesellenvereine) avaient été fondées avant la promulgation de la loi sur la liberté de commerce et d'industrie (1872) et comptaient parmi les premières associations des Roumains pendant la période du Compromis austro-hongrois, les regroupements d'artisans et d'entrepreneurs germanophones (Gewerbevereine) fondés au début des années 1840 faisaient partie des associations les plus anciennes et les plus influentes de toute la Transylvanie ${ }^{(3)}$, tant

* Stéphanie Danneberg a fait ses études, entre autres, à l'Institut d'études politiques de Lille et au Centre d'études germaniques de Strasbourg. Elle a été collaboratrice de projet scientifique jusqu'en juillet 2017 à l'Institut für deutsche Kultur und Geschichte Südosteuropas e. V. an der Ludwig-Maximilians-Universität de Munich, sous la tutelle du prof. Wolfgang Dahmen de la Friedrich-Schiller-Universität Jena.

1 Il s'agit d'un article portant sur un extrait de la thèse de doctorat que Stéphanie Danneberg a soutenue en février 2017 à la Ludwig-Maximilians-Universität de Munich (Historisches Seminar, Geschichte Osteuropas und Südosteuropas) sous la direction du prof. Martin Schulze Wessel. La thèse, qui a été retravaillée en vue d'une publication, est parue en $2018 \mathrm{chez}$ Vandenhoeck \& Ruprecht (Göttingen) sous le titre: Wirtschaftsnationalismus lokal. Abgrenzung und Interaktion zwischen rumänischen und sächsischen Gewerbeorganisationen in den siebenbürgischen Zentren Hermannstadt und Kronstadt, 1868-1914, dans la collection «Schnittstellen. Studien zum östlichen und südöstlichen Europa» que dirigent les professeurs Ulf Brunnbauer et Martin Schulze Wessel à la Graduiertenschule für Ost- und Südosteuropastudien de Munich.

2 Revista Economică, n 34 (22.08.1903), «Exposiția industriaşilor din Sibiiu» (exposition industrielle de Sibiu) : «Este o exposiție frumoasă, de mare interes nu numai pentru exposanți şi pentru poporul săsesc în genere, ci foarte instructivă şi pentru meseriaşii noştri şi pentru poporul românesc.»

3 La Transylvanie (en allemand Siebenbürgen, en roumain Ardeal ou Transilvania, en hongrois Erdély), dont il est question ici, et qui aujourd'hui fait partie intégrante de l'État roumain, correspond non pas à la Transylvanie au sens large qui comprend généralement le Banat et les différents territoires du Partium (Partium regni Hungariae), mais à son sens restreint, la Transylvanie historique correspondant au Siebenbürgen allemand. 
au niveau du nombre de membres que de leur patrimoine et de l'influence exercée localement et régionalement. L’association Hermannstädter Bürger- und Gewerbeverein $^{(4)}$ fondée en 1840 à Hermannstadt (Sibiu en roumain, nom actuel de la ville de Nagyszeben en hongrois) par les Saxons ou groupe germanophone de Transylvanie ${ }^{(5)}$, nomme dans sa brochure commémorative, publiée à l'occasion de son cinquantième anniversaire, deux domaines dans lesquels elle concentrait ses activités: la formation et l'organisation d'expositions artisanales (dites «industrielles»): «zur unmittelbaren Unterstützung der Gewerbsthätigkeit» ${ }^{(6)}$. L'exposition artisanale et industrielle (Gewerbeausstellung) était un médium de représentation et de "mise en scène» de l'économie «nationale» très prisé au XIX ${ }^{\mathrm{e}}$ siècle et donc un instrument puissant entre les mains des nationalistes ${ }^{(7)}$. L'analyse de ces expositions locales permet de comprendre la culture économique des Saxons, des Roumains et des Magyars (les trois groupes nationaux les plus nombreux de la Transylvanie) et met en évidence une relation roumano-saxonne résolument différente dans les deux villes transylvaines Hermannstadt et Kronstadt (Braşov, nom actuel; Brassó, en hongrois); différence dans laquelle les Magyars (en tant que Tertium Comparationis) jouaient un rôle prépondérant. Ces derniers étaient devenus, à l'issue du Compromis austro-hongrois, nation d'État face aux «nationalités» (les populations déclarant une langue maternelle autre que le hongrois, donc les groupes non magyars) saxonne et roumaine ${ }^{(8)}$. Les Magyars se considéraient

4 L'histoire de la fondation de l'association d'artisans saxons Hermannstädter Bürger-und Gewerbeverein est peu connue. On sait que le premier comité de direction fut élu le 6 juillet 1840 à l'issue d'une assemblée réunissant 189 membres. Le premier président élu était Joseph Benigni von Mildenberg (1782-1849), ancien «k.k. Feldkriegssekretär» à la frontière militaire transylvaine (siebenbürgische Militärgrenze) et rédacteur du journal transylvain germanophone Siebenbürger Boten.

5 Les statistiques portant sur la population germanophone ne permettent pas toujours de faire une distinction précise entre les différents types de groupes allemands en Transylvanie. Population germanophone: «saxonne» et luthérienne, ou, à la suite de différentes phases d'immigration, allemande et catholique d'Allemagne, d'Autriche ou encore de la zone Bohême/Moravie/Silésie. Citons par exemple Rolf Kutschera sur l'installation de fonctionnaires allemands catholiques dans la ville de Kronstadt aux XVIII ${ }^{\mathrm{e}}$ et XIX ${ }^{\mathrm{e}}$ siècles ou encore à la suite de la révolution de 1848/1849: "Bei den Amtskandidaten handelte es sich entweder um zugezogene Österreicher oder Böhmen, die ihre katholische Konfession quasi als Empfehlungsschreiben, "um in den Magistrat gezogen zu werden" mitbrachten, oder um Kronstädter bzw. Burzenländer, die aus materiellen Gründen zur katholischen Konfession konvertierten.» Rolf Kutschera, «Kronstadt während der Regierungszeit der Habsburger (1688-1918)», in: Harald Rотн (dir.), Kronstadt: Eine siebenbürgische Stadtgeschichte, Munich, Universitas, 1999, p. 61.

6 Julius Hann von Hannenheim, Der Hermannstädter Bürger- und Gewerbeverein. 1840-1890. Zur Feier des fünfzigjährigen Bestandes, Hermannstadt, Krafft, 1890: «comme support direct de l'activité artisanale».

7 Voir Thomas Grossbölting, «Im Reich der Arbeit»: Die Repräsentation gesellschaftlicher Ordnung in den deutschen Industrie- und Gewerbeausstellungen 1790-1914, Munich, R. Oldenbourg, 2008, p. 23 : «nationaler Selbstdefinition und Selbstrepräsentation».

8 Après plusieurs siècles de dominations successives des Hongrois, des Ottomans et des Habsbourg, la Transylvanie fut intégrée au royaume de Hongrie en 1868 par la loi d’union XLIII: 1868. La constitution de mars/avril 1848 avait proclamé l’Union de la Principauté de Transylvanie avec la Hongrie: le rétablissement de cette constitution et donc de l'Union était une condition sine qua non des négociations austro-hongroises (1865-1867) qui aboutirent au "Compromis» (Ausgleich) et transformèrent l'Empire d'Autriche en double monarchie austro-hongroise reposant sur une union personnelle par la maison de Habsbourg-Lorraine. La loi sur les nationalités (loi XLIV: 1868) proclama l'égalité de droits de tous les citoyens du royaume de Hongrie et donc mit pour la première fois les Roumains 
en Transylvanie comme étant la seule nation historique, donc les seuls ayant une existence politique et une tradition d'État. Ils considéraient l'édification de l'État national hongrois comme «aboutissement de l'histoire de la Hongrie » ${ }^{(9)}$.

Le Bürger- und Gewerbeverein saxon avait organisé entre 1840 et 1914 de nombreuses expositions artisanales à Hermannstadt, dont celle de 1869 qui mérite une attention particulière ${ }^{(10)}$. Cette première grande manifestation attira en quelques jours plus de 4000 personnes et généra un bénéfice de 1535 florints (fl.): ce qui était à l'époque et pour une ville de taille moyenne du sud de la Transylvanie - région essentiellement agricole à la périphérie de l'Empire des Habsbourg - un résultat impressionnant ${ }^{(11)}$. Les 171 exposants étaient majoritairement des artisans saxons, membres de l'association Bürger- und Gewerbeverein mais aussi des artisans, compagnons et apprentis d'autres appartenances ethno-culturelles qui avaient répondu à l'appel du comité d'organisation:

«Durch die Gewerbe-Ausstellung wird jedem Erzeuger irgend einer Sache die Gelegenheit geboten, sein Erzeugniß in möglichst weiten Kreisen bekannt zu machen und selbst den Stand und die Fortschritte der Gewerbe kennen zu lernen. [...] es ist daher nicht nothwendig Meister oder Mitglied einer Zunft zu sein, um zur Ausstellung seiner Erzeugnisse berechtigt zu sein ${ }^{(12)}$.

Bien qu'en 1869, la quasi-totalité des corporations de Hermannstadt fût «encore» saxonne ${ }^{(13)}$, le message du comité directeur était adressé à « tous » les citadins (Stadtbürger)

de Transylvanie en situation d'égalité de droits avec leurs concitoyens saxons. Ces derniers avaient constitué l'une des classes dominantes de la Transylvanie (Saxones ou Natio Saxonica), joui d'un statut particulier et de nombreux privilèges, tandis que les Roumains n'avaient eu accès à la citoyenneté qu'en 1863 et n'avaient jamais constitué de natio, au sens médiéval du terme.

9 Joachim von Puttк Amer, Schulalltag und nationale Integration in Ungarn. Slowaken, Rumänen und Siebenbürger Sachsen in der Auseinandersetzung mit der ungarischen Staatsidee 1867-1914, Munich, R. Oldenbourg, 2003, p. 37 : «Vollendung der Geschichte Ungarns».

10 Biblioteca Brukenthal: fonds «Manuscrise»/«probleme de expoziții meșteșugărească» (fonds «Hermannstädter Bürger- und Gewerbeverein»), nº 380 (1869), doc. 221-224/1.

11 Hermannstadt comptait 18998 habitants en 1869.

12 Biblioteca Brukenthal: fonds «Manuscrise »/«probleme de expoziții meşteşugărească », $n^{\circ} 380$ (1869), doc. 221-224/1: «Grâce à l'exposition artisanale, tout fabricant quel qu'il soit aura la possibilité de présenter sa production à un large public et également d'évaluer le niveau actuel de développement de l'artisanat et ses progrès. Il n'est donc pas nécessairement obligatoire d'être maître-artisan ou membre d'une corporation pour être autorisé à exposer.»

13 En Transylvanie, l'acquisition de terres sur le territoire saxon (Königsboden ou Fundus regis), tout comme l'accès au droit de cité dans les villes (intra-muros) de ce territoire, étaient réservés exclusivement aux Saxons. Ces droits furent remis en question par le rescrit de l'empereur Joseph II en 1781 (Konzivilitätsreskript) qui autorisait désormais les membres des autres «nations» à acquérir des terres sur ce territoire. Le Deutschtum n'était en outre plus considéré comme condition sine qua non du droit à la résidence (Bürgerrecht) dans les villes situées sur le Königsboden. Les non-Saxons étaient donc jusqu'en 1781 personae non gratae à l'intérieur de ces villes; ce qui concernait autant le groupe ethnoculturel des Roumains (qui ne jouissaient d'aucun droit civique et politique) que celui des Magyars. Afin de protéger leur monopole dans l'artisanat et les corporations, les Saxons introduisirent l'obligation de fournir un "Taufschein» qui permettait exclusivement aux protestants luthériens, donc aux Saxons, d'avoir accès au système corporatif. Les Saxons purent de cette manière continuer à dominer l'artisanat jusqu'à l'introduction de la liberté de commerce et d'industrie en 1872. 
de Hermannstadt, sans distinction d'appartenance «nationale» ou religieuse. L'intégration économique de la Transylvanie au royaume de Hongrie et à la zone douanière de l'Empire austro-hongrois dès la fin de la révolution de 1848-1849 avait, entre autres, permis l'introduction de la liberté de commerce et d'industrie (Gewerbefreiheit) en deux étapes, en 1859 puis en 1872; condition sine qua non à une modernisation économique et sociale du territoire. Ces conditions «imposées» depuis Vienne et Budapest étaient moins un signe d'impérialisme que le revers de l'incorporation d'une zone géographique économiquement en retard au grand marché que constituait l'Autriche-Hongrie. La liberté de commerce ouvrit la voie dans les villes saxonnes, et particulièrement à Hermannstadt et Kronstadt, à une concurrence sans précédent entre Magyars, Saxons et Roumains, car le système corporatif maintenu jusqu'en 1872 avait jusque-là permis aux seuls Saxons de protéger leur monopole dans l'artisanat. Pourtant, malgré la concurrence économique exacerbée depuis 1872, la deuxième grande exposition artisanale organisée par le Bürger- und Gewerbeverein en 1903 attira jusqu'à 27309 visiteurs et 271 exposants : parmi eux, un grand nombre de compagnons et apprentis non germanophones, surtout roumains, dont certains obtinrent médailles et récompenses pour les objets exposés ${ }^{(14)}$. Des articles très intéressants portant sur cette manifestation organisée par l'association saxonne sont autrefois parus dans la revue économique roumaine Revista Economică dont le siège de la rédaction se trouvait à Hermannstadt. Les articles allaient de l'invitation de l'association, adressée aux artisans de la ville, à un compte-rendu détaillé de l'exposition:

"C'est une belle exposition, pas seulement d'un grand intérêt pour les Saxons mais pour tous les citadins. Elle est également très instructive pour nos artisans et pour le peuple roumain. C'est la raison pour laquelle elle mérite d'être visitée par tous ceux qui s'intéressent au développement de l'industrie et à celui de l'économie des peuples en général » ${ }^{(15)}$.

La revue n'avait pas seulement reproduit l'ensemble du discours de la cérémonie d'ouverture mais en approuvait clairement certaines déclarations, dont celle portant sur la nécessité de pilotage du comportement d'achat des habitants de la ville de Hermannstadt. Les associations, entre autres d'artisans et d'industriels, avaient pour mission d'inciter leurs "conationaux" à n'acheter que "local», un argument majeur de la rhétorique du nationalisme économique, ici, en Transylvanie et, à l'époque, dans toute l'Europe centrale ${ }^{(16)}$.

L'artisanat roumain de Hermannstadt était représenté depuis 1867 par l'Association des compagnons roumains de Sibiu (Reuniunea sodalilor români din Sibiiu) ${ }^{(17)}$. L'association parvint à organiser successivement en 1892 et 1902 deux expositions locales d'artisans roumains, dont l'écho particulièrement positif lui offrit popularité

14 Kronstädter Zeitung, $\mathrm{n}^{\circ} 219$ (24.09.1903); Biblioteca Brukenthal: fonds «Manuscrise»/«probleme de expoziții meşteşugărească ", nº 405 (1903), doc. 1-32.

15 Revista Economică, n 34 (22.08.1903), «Exposiția industriaşilor din Sibiiu» (exposition industrielle de Sibiu).

16 Ibid.: «[...] şi ca consumenții să spriginească produsele industriale din loc vrednice de spriginit».

17 L'archiprêtre orthodoxe roumain Nicolae Cristea (1834-1902) a été en 1867 l'initiateur de la fondation de l'association Reuniunea sodalilor români din Sibiiu Proche du métropolite de l'Église orthodoxe de Transylvanie, l’aroumain Andrei Șaguna (1809-1873), il était à l'époque une personne publique très influente. 
et notoriété dans toute la région transylvaine. Malgré des moyens financiers restreints et un nombre d'exposants modeste, on attendait de la première exposition industrielle des Roumains de la ville qu'elle puisse servir d'exemple et stimuler les artisans roumains de toute la Transylvanie:

«Voilà pourquoi nous devons mettre tout notre cœur à l'ouvrage, pour que cette première entreprise puisse être reproduite par d'autres associations d'artisans roumains, à Braşov, à Cluj et dans toutes les autres villes où travaillent nos artisans» ${ }^{(18)}$.

L'exposition roumaine de 1892 attira 735 visiteurs et regroupa 69 exposants: il s'agissait essentiellement de compagnons et apprentis roumains, mais travaillant dans des ateliers qui appartenaient surtout à des Saxons. La composition ethno-nationale de l'artisanat et de l'industrie était la suivante à Hermannstadt ${ }^{(19)}$ :

\begin{tabular}{|c|c|c|c|c|c|}
\hline $\begin{array}{c}\text { Ateliers/ } \\
\text { manufactures: }\end{array}$ & \multicolumn{2}{|c|}{1878} & \multicolumn{2}{|c|}{1911} & $\begin{array}{l}\text { Croissance } \\
1878-1911\end{array}$ \\
\hline Allemands & 407 & $74,3 \%$ & 751 & $58,0 \%$ & $84,0 \%$ \\
\hline Roumains & 8 & $1,5 \%$ & 182 & $14,0 \%$ & $2175,0 \%$ \\
\hline Magyars & 29 & $5,3 \%$ & 121 & $9,3 \%$ & $317,2 \%$ \\
\hline Juifs & 18 & $3,3 \%$ & 70 & $5,4 \%$ & $288,0 \%$ \\
\hline Autres & 86 & $15,7 \%$ & 172 & $13,3 \%$ & $100,0 \%$ \\
\hline Total & 548 & $100,0 \%$ & 1296 & $100,0 \%$ & $136,5 \%$ \\
\hline
\end{tabular}

La différence principale entre les ateliers des Roumains et ceux des autres groupes consistait dans la taille de l'entreprise: les artisans roumains travaillaient généralement seuls, tandis que Saxons (surtout) et Magyars employaient et formaient des apprentis et des compagnons. Le renouvellement de génération dans les ateliers saxons s'opérait souvent par les Roumains, puisque l'artisanat était devenu en 1911, notamment pour la jeune génération saxonne, un secteur beaucoup moins lucratif. Cette situation explique qu'en 1892, le jury de l'exposition artisanale roumaine, qui était chargé d'émettre des avis sur les objets artisanaux exposés, se composait de douze maîtres-artisans représentant la plupart des nationalités de la ville de Hermannstadt, surtout allemande et saxonne ${ }^{(20)}$. Cela concernait également l'exposition roumaine de

18 Direcția Județeană Sibiu a Arhivelor Naționale: fonds Reuniunea meseriașilor români din Sibiu (anii 1867-1948), dos. 1 (1882-1900), doc. 28 (1891): «De aceea trebue se îmbrățişăm acéstă causă cu tótă căldura inimei, căci de sigur acéstă încercare a nóstră va fi imitată și de celealte reuniuni de meseriași români, din Brașov, Cluş și alte orașe, pe unde sunt meseriași români. »

19 Voir: Tabelle 12. Ethnische Zusammensetzung des Handwerkswesens in Hermannstadt, 1878-1911, in: Stéphanie Danneberg, Wirtschaftsnationalismus lokal (note 1). C’est la première démonstration statistique de la composition ethno-nationale de l'industrie à Hermannstadt. Seuls les chiffres portant sur l'artisanat et l'industrie de Kronstadt avaient jusqu'à maintenant été publiés.

20 Voir: Anuarul I. al Reuniunii sodalilor români din Sibiu cuprinzând unele date dela întemeierea ei pâna la 31 decembrie 1899 (annuaire de l'Association des compagnons roumains de Sibiu, comprenant des événements depuis sa fondation jusqu'au 31 décembre 1899), Sibiu, 1900, p. 24; Adressbuch der k. freien Stadt Hermannstadt, VIII. Jahrgang, Hermannstadt, W. Krafft, 1898. Le jury se composait de: Nicolae Simtion, imprimeur; Karl Bell, boucher (?); Johann Szentgyörgyi, cordonnier; Ludwig Szántó, cordonnier; Valentin Zinz, cordonnier; Karl Theil, serrurier; Johann Borthmes, menuisier; Samuel Reinerth, tanneur; Francis A. Wensky, tailleur; Ludwig Ilyési, tapissier; Eduard Rannicher, maroquinier; Johann Keil, boulanger. 
1903 dont le jury, composé de trente membres, était représentatif de tous les groupes de population de Hermannstadt; il fut aussi convié aux festivités organisées par l'association «Reuniunea» à l'issue de la remise des médailles pour les objets primés ${ }^{(21)}$. Le comité directeur de l'association avait reçu à cette occasion le soutien du journal saxon Siebenbürgisch-Deutsches Tageblatt qui avait publié à plusieurs reprises l'appel du comité roumain aux artisans de la ville:

«Es wird in romänischen Kreisen gewünscht und wir geben dem Ausdruck dieses Wunsches bereitwillig Raum, daß unsere Gewerbetreibenden, die in ihren Werkstätten in Verwendung stehenden romänischen Gesellen und Lehrlinge dazu anregen mögen, sich an dieser Ausstellung mit Erzeugnissen ihres Fleißes zu beteiligen » ${ }^{(22)}$.

À Kronstadt, le comité directeur de l'«Association de soutien des compagnons et apprentis roumains de Braşov» ("Asociația pentru sprijinirea învățăceilor şi sodalilor români din Braşov») ${ }^{(23)}$, fondée en 1869, avait jusqu'à la veille de la Première Guerre mondiale persisté dans son refus d'organiser la moindre exposition, ce que le journal roumain Gazeta Transilvaniei commentait régulièrement:

"Quand allons-nous, Roumains, fonder de telles associations, pour que nous puissions nous aussi organiser de pareilles expositions? Et qui plus est, ils [les Saxons] ne tiennent aucune assemblée générale sans organiser une quelconque exposition industrielle ou un salon de l'agriculture ${ }^{(24)}$.

Les artisans saxons de Kronstadt regroupés, eux, au sein du Kronstädter Gewerbeverein $^{(25)}$, fondé en 1841, rejetaient les expositions artisanales régulières qu'ils

21 Fonds Reuniunea meseriașilor români din Sibiu (anii 1867-1948), dos. 3 (1903-1905), doc. non répertorié/numéroté, "Raport General despre esposiția industrială românească din a. 1902» (rapport portant sur l'exposition industrielle roumaine de l'année 1902). Le fonds d'archives de l'association des artisans roumains de Hermannstadt n'est pas accessible au public. Il n'est que partiellement numéroté et n'a pas été répertorié jusqu’à ce jour.

22 Siebenbürgisch-Deutsches Tageblatt, $\mathrm{n}^{\circ} 8737$ (13.09.1902): «Les milieux roumains de la ville souhaitent, et c'est une demande que nous soutenons volontiers, que nos artisans qui emploient des compagnons et apprentis roumains, incitent ces derniers à participer à cette exposition avec les produits artisanaux qu'ils ont fabriqués.»

23 Le prêtre orthodoxe roumain Bartholomäu (Bartolomeu) Baiulescu (1831-1909) est à l'origine de la fondation de l'Association de soutien des compagnons et apprentis roumains de Braşov. Tout comme Cristea, Baiulescu était un proche du métropolite orthodoxe Andrei Șaguna et une personne publique. Il était proche des milieux artistiques et intellectuels roumains et avait des liens étroits avec les familles des grands commerçants roumains de Kronstadt. Celles-ci avaient constitué, avant leur déclin dans la seconde moitié du XIX ${ }^{\mathrm{e}}$ siècle, la bourgeoisie riche et influente de Kronstadt («Die große Masse der sächsischen Bürgerschaft lebte damals in sehr bescheidenen Vermögensverhältnissen, die reichsten kapitalkräftigsten Leute waren die rumänischen Großkaufleute», Heinrich WACHNER, Kronstädter Heimat- und Wanderbuch, Kronstadt-Brasov, Wilh. Hiemesch, 1934, p. 288).

24 Gazeta Transilvaniei, nº 49 (18./06.07.1874): «Reuniunea economică a saşiloru din Transilvani’a va tine in dilele dela 20.23 Augustu a. c. o adunare generale in Brasiovu, cu care occasiune se va arangiá si o espusetiune economică. [...] òre noi romanii, candu vomu avé Reuniuni economice, cá se potemu arangia si espusetiuni de acésta natura? [...] Mai multu nece o adunare a Asociațiunei se nu treaca fără expusetiunea vreunui ramu de cultură industriale séu economică-agricolă.»

25 Kronstädter sächsischer Gewerbeverein, 100 Jahre heimat- und volksverbundene Gewerbevereinsarbeit in Kronstadt, Kronstadt, 1941, p. 17: les initiateurs de l'association ont été le sénateur et économiste saxon Peter Traugott Lange von Burgenkron (1797-1875), l'imprimeur allemand (né en Allemagne 
qualifiaient de «Ausstellungsfieber»(26), critiquant la fréquence trop soutenue des expositions de Hermannstadt. La seule exposition importante de la ville de Kronstadt avait été celle d'août 1886, mais fut l'objet de nombreuses controverses, ce que déplorait la rédaction du Siebenbürgisch-Deutsches Tageblatt de Hermannstadt: «Es ist doch außerordentlich betrübend, daß die nationalen und konfessionellen Hetzer auch in wirtschaftlichen Dingen die Gemüter gegeneinander treiben » ${ }^{(27)}$. Quelques jours seulement avant le début de la manifestation, la plupart des exposants roumains, mais aussi magyars, annulèrent leur participation, une action que la revue économique roumaine Meseriaşul Român avait commentée comme suit: "Ils [les artisans roumains] ont finalement décidé de ne pas participer à l'exposition. Même les Magyars de Kronstadt ont refusé d'exposer, parce que l'exposition se présente comme étant celle des entrepreneurs saxons ${ }^{(28)}$.

Il s'agit donc ici de deux villes de la même région, séparées par moins de deux cents kilomètres, dans lesquelles la thématique de l'organisation d'expositions artisanales donne à voir des relations interethniques profondément différentes. Dans le centre urbain de Hermannstadt, les relations consensuelles entre Saxons et Roumains, aussi bien dans la presse locale qu'entre les principaux acteurs, les artisans et les comités d'organisation et (ou) directeurs d'associations d'artisans, ont eu une action bénéfique sur l'organisation d'événements qui profitaient à la ville et à tous ses habitants. En revanche, le clivage ethno-national de Kronstadt n'avait pas abouti à un consensus sur l'organisation d'une exposition artisanale réussie et représentant les divers groupes nationaux de la ville.

Hermannstadt et Kronstadt occupaient parmi les centres historiques germanophones de la Transylvanie une place tout à fait particulière. Hermannstadt avait été longtemps la capitale politique de la région puisque c'est là que siégeait depuis le Moyen Âge l'Université saxonne (sächsische Nationsuniversität), la plus haute instance politique, administrative et judiciaire des Saxons. De même (re)devint-elle en 1867 la capitale confessionnelle de ces derniers puisque l'évêque de l'Église protestante luthérienne (evangelische Kirche Augsburgischer Bekenntnisses) y résidait. C’est

et immigré en Transylvanie) et futur maire de Kronstadt, Johann Gött (1810-1888), l'artisan lainier Michael Traugott Kamner, l'instituteur (?) Samuel Schiel (1812-1881 ?) et le commerçant et futur homme politique, Carl Maager (1813-1887). C'est le 28 novembre 1841, à l'issue de la réunion de 87 artisans et trois instituteurs à l'Hôtel Numéro 1 de Kronstadt, que l'association fut créée.

26 Ibid., p. 108: «[...] denn mehr oder weniger gehöre auch ich und die Mehrzahl der Mitglieder dieses Gewerbevereins zu denjenigen, welche eine zu oft eintretende Wiederkehr von größeren Ausstellungen schon aus dem Grunde nicht billigen, weil solche nicht nur dem Lande, wo dieselben stattfinden, sondern auch den einzelnen Teilnehmern verhältnismäßig große materielle Opfer auferlegen und eben infolge dieser großen Opfer fast nur der Großindustrie zugänglich sind. Es soll nicht eine Ausstellung sein mit großen Opfern des Staates oder der Stadt und des Einzelnen, mit den Preisen und Auszeichnungen und dadurch auch den Ehrgeiz weckenden Konkurrenzkämpfen, kurz nicht mit den Mitteln und Zielen einer großen Ausstellung [...].»

27 Siebenbürgisch-Deutsches Tageblatt, $\mathrm{n}^{\circ} 3854$ (18.08.1886): «Il est particulièrement affligeant de constater que les agitateurs nationalistes et religieux parviennent à attiser les conflits jusque sur le terrain de l'économie.»

28 Meseriaşul Român, $\mathrm{n}^{\circ} 12(1 . / 13.08 .1886)$ : «Aşa s’au hotărit a nu espune. Maghiarii meseriaşi de aci incă s'au oprit a espune, pentrucă esposiția apare a asociațiunei industriaşilor Saşi din Braşov. » 
au début du $\mathrm{XX}^{\mathrm{e}}$ siècle que Hermannstadt devint aussi la capitale culturelle et confessionnelle des Roumains de Transylvanie avec l'inauguration du musée («national») roumain Astra en 1905, suivie en 1906 de celle de la cathédrale orthodoxe roumaine, un événement à caractère hautement symbolique. La ville de Kronstadt, quant à elle, avait préservé pendant le Moyen Âge son rang de haut bourg du commerce transylvain et lieu de passage stratégique entre les commerces d'Orient et d'Occident. La ville conserva ce statut de ville marchande, même lorsque Hermannstadt commença à lui faire concurrence. La relation étroite entre les deux centres transylvains fut marquée par une concurrence économique et politique importante qui concerne aussi la période que nous analysons ici (1868-1914); une concurrence qui se retrouve dans les relations roumano-saxonnes, entre les Saxons de Hermannstadt et de Kronstadt mais aussi entre les Roumains des deux villes. Une comparaison entre les deux centres transylvains est également pertinente au regard des structures démographiques, très différentes: tandis que Hermannstadt resta une ville à majorité allemande ou «saxonne» - d'après le dernier recensement hongrois datant de 1910 -, Kronstadt, elle, se «magyarisa » ${ }^{(29)}$. Ces différences démographiques (par naissance, immigration et assimilation) furent à l'origine de conflits d'intérêts économiques et politiques distincts dans les deux centres. À Kronstadt, avant la Première Guerre mondiale, les Magyars

«[...] composaient la majorité relative de la population, avaient atteint dans l'artisanat un niveau de développement aussi élevé que celui des Allemands, dominaient une grande partie du secteur tertiaire et de l'administration publique et, enfin, prenaient, avec les Allemands, les décisions politiques, surtout au niveau régional» ${ }^{(30)}$.

Si les Saxons dominaient encore, en 1911, l'artisanat et l'industrie à Hermannstadt, et que les Roumains affichaient une croissance de plus de $2000 \%$ entre 1878 et 1911, comme le montre le tableau ci-dessus, les Magyars dominaient, eux, l'artisanat de Kronstadt puisque 44,6 \% des ateliers de la ville leur appartenaient. Les autres ateliers se répartissaient entre les germanophones (Saxons et Allemands catholiques) avec $35,4 \%$, les Roumains avec $16,5 \%$ et les juifs avec 3,6 \% ${ }^{(31)}$. Les Saxons se trouvaient

29 D'après le dernier recensement hongrois de 1910, Kronstadt comptait 41056 habitants : 17831 Magyars (43,4 \%), 11786 Roumains (28,7\%), 10841 Allemands (26,4\%) et 598 ou 1,4\% de personnes d'autres appartenances ethno-culturelles (Arméniens, Grecs, Bulgares, Ruthènes, etc.). À cela venaient s'ajouter environ 1417 habitants juifs (3,4\%) qui se déclaraient majoritairement soit Allemands (de langue allemande) ou Magyars (de langue hongroise). À Hermannstadt vivaient en 191033489 habitants, dont 16832 Allemands (50,3 \%), 8824 Roumains (26,3 \%), 7252 Magyars (21,6 \%) et 581 personnes ou $1,7 \%$ d'autres appartenances. Les 33489 habitants comptaient environ 1307 juifs (3,9\%).

30 Gerald Volkmer, «Gründerzeit im Karpatenbogen - das siebenbürgische Burzenland und die Herausforderungen der Industrialisierung 1867-1918», in: Matthias Weber (dir.), Gründerzeit, Jahrbuch des Bundesinstituts für Kultur und Geschichte der Deutschen im Östlichen Europa, vol. 21, Munich, Oldenbourg, 2013, p. 66: «[...] stellten die relative Bevölkerungsmehrheit, hatten sich eine den Deutschen vergleichbare starke Stellung im Handwerk erarbeitet, dominierten große Teile des Dienstleistungsbereichs und der öffentlichen Verwaltung und bestimmten zusammen mit den Deutschen die politischen Geschicke, vor allem auf der Komitatsebene.»

31 Voir: Tabelle 10. Ethnische Zusammensetzung des Handwerkswesens in Kronstadt 1860-1911 und Vergleich mit den Zahlen des Kronstädter Gewerbevereins 1912, in: S. DANNEBERG, Wirtschaftsnationalismus lokal (note 1). Il s'agit ici précisément des chiffres de Friedrich Lexen pour l'année 
surtout au premier plan de la création d'entreprises industrielles ou de la reconversion de maîtres-artisans en fabricants, même si, ici, à la périphérie de l'Empire, la Transylvanie ne connut pas, à proprement parler, de révolution industrielle. Les grands perdants du développement économique étaient donc, à Kronstadt, les Roumains.

La thématique de l'organisation d'expositions met en lumière le clivage ethno-national difficilement surmontable à Kronstadt: la concurrence économique fut instrumentalisée et propagée comme «conflit ethno-national». L'exposition artisanale était à Kronstadt un «lieu de polarisation nationale». À Hermannstadt l'organisation régulière d'expositions d'artisans avait été rendue possible par le consensus et la coopération entre les divers groupes ethno-culturels, notamment entre Saxons et Roumains; l'exposition permettait ici une visualisation positive de l'économie locale et de ses acteurs.

Une analyse plus approfondie de la rhétorique nationaliste intervenant au centre de l'organisation (ou de la non-organisation) des expositions dévoile d'autres aspects de la culture économique des Saxons et des Roumains: "Au-delà de l'aspect économique, les expositions artisanales avaient pour vocation de générer des émotions: artisanat et industrie devaient se présenter comme vecteur et catalyseur de la "fierté nationale" » ${ }^{(32)}$. Catherine Albrecht, qui a analysé la grande exposition générale de Prague de 1891 (jubilé de la première exposition industrielle de 1791), avait également démontré le rôle catalyseur de l'exposition chez les Tchèques en Bohême: «The young Czechs supported a national exhibition emphasizing not just the economic achievements of the Czechs but the whole cultural progress of the Czech nation ${ }^{(33)}$. Les expositions des Saxons de Hermannstadt étaient axées sur la mise en valeur de l'économie locale (au profit de «tous») et la libre concurrence, représentée par une remise de prix dans laquelle l'habileté de l'artisan comptait plus que la préférence «nationale». La manifestation de 1886 organisée par le Kronstädter Gewerbeverein mettait, elle, en avant une économie «nationale» saxonne centrée sur la tradition (« um "auch einen Blick in die Vergangenheit" zu richten») ${ }^{(34)}$, la communauté («auf das "gemeinsame Streben" und den "Nutzen der Gemeinschaft", [...] nach innen hin und unter uns selbst») (35) et une certaine nostalgie du système corporatif. Contrairement aux expositions de commerce et d'industrie de l'époque pendant lesquelles concours et remises de prix revêtaient une grande importance, on avait renoncé à Kronstadt à ce moment symbolique: c'est justement la concurrence qui était le moteur de l'économie moderne et du capitalisme que l'on rejetait ici.

1912 (Friedrich Lexen, «Zur wirtschaftlichen Entwicklung Kronstadts», Kronstädter Zeitung, n 78 [04.04.1912]).

32 S. DAnneberg, ibid.: «[Ü]ber den wirtschaftlichen Aspekt hinaus sollten die Gewerbeausstellungen eine "emotionale" Wahrnehmung bewirken: Handwerk und Industrie sollten als Träger und Katalysator des "Nationalstolzes" fungieren.».

33 Catherine Albrecht, «Pride in Production: The Jubilee Exhibition of 1891 and Economic Competition between Czechs and Germans in Bohemia», Austrian History Yearbook, 24 (1993), p. 102.

34 S. DANNeBerg, Wirtschaftsnationalismus lokal (note 1), cité d'après 100 Jahre Gewerbevereinsarbeit in Kronstadt, p. 109: «afin d'orienter les regards vers le passé».

35 Ibid.: «aux "efforts communs" et à la "nécessité de la communauté", au cœur de nous-mêmes et entre nous». 
L'analyse du nationalisme économique «intra-étatique» («un paradigme tombé en disgrâce ») ${ }^{(36)}$, dans l'espace transylvain, ici basé sur l'exemple d'expositions organisées par les associations d'artisans ou de compagnons, se place dans la suite de recherches auparavant menées par Catherine Albrecht, citée plus haut, ou celles menées entre 2001 et 2005 à l'Europa-Universität Viadrina à Francfort-sur-l'Oder sous la direction de Helga Schultz. À l'issue du projet de Schultz, des études majeures portant surtout sur la République tchèque (Bohême, Moravie et Silésie) et la Tchécoslovaquie, la Pologne (Galicie) et la Hongrie ont été publiées ${ }^{(37)}$. Cependant, une analyse du nationalisme économique transylvain basée sur les associations d'artisans et de compagnons dont la fondation remonte au milieu du XIX ${ }^{\mathrm{e}}$ siècle est une approche particulièrement novatrice, tant au niveau de la thématique que de la période choisie.

\section{Le concept du nationalisme économique «intra-étatique» en Europe centrale}

La méthode adoptée ici est certes celle d'une micro-histoire locale, mais toujours en lien étroit avec le phénomène du nationalisme économique observable dans d'autres régions de l'Empire des Habsbourg et dans l'Europe centrale et orientale. Le nationalisme économique, conçu ici comme «intra-étatique» et propre à de nombreuses régions de l'Europe centrale de l'entre-deux-guerres, valait également pour l'espace transylvain d'avant la Première Guerre mondiale. Cette analyse du nationalisme est donc particulière puisque la recherche sur le nationalisme économique est généralement «internationale», allant de l'entre-deux-guerres à nos jours. Le nationalisme économique se rapporte dans sa forme intra-étatique (innerstaatlicher Wirtschaftsnationalismus) à une pratique ou culture économique entre différents groupes ethnoculturels à l'intérieur d'un État ou d'une région, ou bien à celle d'un groupe minoritaire en opposition à une majorité ethnique différente et souvent au pouvoir. Citons parmi les définitions de cette forme particulière du nationalisme économique, celle d'Ágnes Pogány: «Le nationalisme économique est une idéologie qui cherche à mobiliser les masses sur fond d'appartenance ethno-culturelle et qui, en outre, aspire à construire la nation non pas seulement culturellement mais aussi économiquement " ${ }^{(38)}$. Les Saxons avaient des intérêts politiques et économiques ancestraux aussi bien à Hermannstadt qu'à Kronstadt, intérêts qu'ils souhaitaient préserver, tandis que les Roumains aspiraient, eux, à une «nation» forte qui, en principe, venait à peine d'émerger; même si une partie de leur élite, aussi infime fût-elle, avait déjà conscience d'une appartenance «nationale» fortement liée à la langue et la confession depuis la fin du XVIII siècle $^{(39)}$.

36 Helga Schultz citée par Ágnes PogÁNY, «Wirtschaftsnationalismus in Ungarn im 19. und im 20. Jahrhundert», in: Ágnes PogÁny, Eduard Kubů, Jan Kofman (dir.), Für eine nationale Wirtschaft. Ungarn, die Tschechoslowakei und Polen vom Ausgang des 19. Jahrhunderts bis zum Zweiten Weltkrieg, Berlin, Berliner Wissenschafts-Verlag, 2006, p. 11 : «ein vergessenes Paradigma».

37 Les historiens impliqués dans le projet de Helga Schultz étaient, entre autres, Eduard Kubů, Torsten Lorenz, Uwe Müller, Rudolf Jaworski et Ágnes Pogány.

38 Á. PogÁNy, «Wirtschaftsnationalismus in Ungarn im 19. und im 20. Jahrhundert» (note 36), p. 13: «Wirtschaftsnationalismus ist eine Ideologie, die versucht Massen anhand ihrer ethnischen Zugehörigkeit zu mobilisieren, und es außerdem anstrebt, die Nation nicht nur kulturell sondern auch wirtschaftlich zu konstruieren.»

39 Seuls les prêtres roumains convertis au gréco-catholicisme avaient accès depuis 1744 aux droits civiques après qu'une partie du clergé orthodoxe roumain s'est «unie » à l'Église catholique romaine 
Le nationalisme économique repose en Transylvanie sur les mêmes éléments mobilisateurs que ceux cités par Helga Schultz dans son projet, à savoir l'arriération (existence de sociétés paysannes encore dominées par l'aristocratie), la domination étrangère (Fremdherrschaft) et la pluralité ethnique, mais aussi sur des éléments propres à la région transylvaine: sa position géographique à la périphérie de l'Empire des Habsbourg, le retard de la modernisation sociale et politique (degré élevé d'analphabétisme, pas ou peu de moyens de transport et de communication, pourcentage infime de la population ayant le droit de vote, etc.), ainsi que celui de l'industrialisation. Le phénomène du nationalisme économique est, d'après Schultz, synonyme de frustrations: quand les inégalités sociales et économiques sont interprétées et articulées comme une injustice «nationale» et quand les «autres» sont tenus responsables de ces inégalités ${ }^{(40)}$. On n'aspirait plus seulement à l'autonomie politique et à la mise en valeur culturelle de la nation (Sprachnationalismus) mais aussi dorénavant au développement ou réajustement de son niveau économique comme méthode supplémentaire de consécration (chez les Saxons) ou de mobilisation (chez les Roumains) de la «nation».

Si les Saxons, et surtout les Roumains, n'avaient pas ou peu les moyens d'influencer la politique au niveau national ou d'y intervenir, puisque le droit de vote en Transleithanie était très restreint et que les Roumains menaient une stratégie de résistance passive, renonçant à envoyer des députés au parlement hongrois, ils étaient localement ou régionalement actifs au travers de leurs coopératives ("as a channel for reaching the masses») (41), de leurs associations d'artisans et (ou) d'industriels et de leurs banques. C'était une méthode de développement économique ethno-nationale qui était propre à la Transylvanie et à de nombreux peuples de l'Europe centrale:

«Politics, the electoral system, and administrative political self-government were the terrain where nationalities felt negative discrimination and frustrations. On the contrary, economic policy driven by "laissez-faire liberalism" and "state non-interventionism" proved to be a favorable playing field for economic competition and permitted self-organization of "non-dominant nations" " ${ }^{(42)}$.

Le nationalisme économique intra-étatique, comme forme particulière du nationalisme, signifie par définition qu'il favorise son propre groupe ethno-national sur le marché économique, ce qui supposait d'avoir conscience d'une appartenance «nationale». Il fallait donc mobiliser les consciences et cette mobilisation ne pouvait se réaliser qu'à travers un processus global d'éducation, à tous les niveaux de la société.

(1699-1701). La vie confessionnelle roumaine de Transylvanie revêtait depuis lors un «caractère biconfessionnel» (d'après Macarie DrAGoI, Orthodox and Greek Catholics in Transylvania (1867-1916): Convergences and Divergences, Yonkers/New York, St. Vladimir's Seminary Press, 2015, p. 19). L'orthodoxie obtint en 1791 le statut de confession «tolérée» de Transylvanie.

C'est le 15 juillet 1863 à Hermannstadt, à l'issue de la première diète (Landtag) autorisée depuis la révolution 1848/1849, que les Roumains de Transylvanie qui n'étaient que "tolérés» obtinrent la reconnaissance et l'égalité de droits civiques avec les autres «nations » de la région.

40 S. Danneberg, Wirtschaftsnationalismus lokal (note 1): "wenn soziale Ungleichheit als nationale Ungerechtigkeit empfunden wird, wenn der "Andere" auf demselben Markt als Konkurrent und Bedrohung interpretiert wird».

41 Attila Hunyadi, «Economic Nationalism in Transylvania», Regio. A review of Studies on Minorities, Politics and Society, 2004, p. 175.

42 Ibid., p. 174. 
En Transylvanie comme en Europe centrale, élites politiques, clergé et comités de direction d'associations, de coopératives et (ou) de banques - il s'agissait de personnes publiques influentes occupant souvent plusieurs de ces fonctions - intervenaient comme mobilisateurs. L'analyse d'autres activités - en dehors donc de l'organisation d'expositions artisanales locales - menées notamment par les associations roumaines de Kronstadt (Asociația depuis 1869) et Hermannstadt (Reuniunea depuis 1867) révèle que le nationalisme économique transylvain avait également une portée culturelle. Les associations d'artisans-compagnons roumains regroupant artisans, compagnons et apprentis ne visaient pas seulement le soutien «économique» mais aussi l'élévation du niveau de culture («nationale») de leurs membres. Tout un éventail de développement culturel était mis à disposition des adhérents, allant du groupe de théâtre à la chorale ou à la déclamation de textes et poèmes, sous-entendu essentiellement roumains.

\section{De l'intérêt d'étudier les associations économiques locales. Du rôle pivot des Magyars}

La culture économique des Saxons et Roumains de Transylvanie et leurs relations sont analysées dans le tissu associatif des centres urbains Hermannstadt et Kronstadt entre 1868 et 1914. Il ne s'agit cependant pas d'une micro-histoire isolée, car ces deux centres périphériques de l'Empire des Habsbourg sont représentatifs de nombreuses autres villes pluriethniques du Vielvölkerreich austro-hongrois. Tels l'historien Pieter M. Judson concernant la monarchie des Habsbourg ou le sociologue Rogers Brubaker dans son étude portant sur la ville transylvaine de Cluj-Napoca ${ }^{(43)}$, l'analyse donne à voir des activistes «nationalistes»- ou selon Brubaker des «entrepreneurs ethno-politiques» ${ }^{(44)}$ - se profilant comme représentants des intérêts du groupe ethno-national auquel ils appartiennent sans pour autant parvenir à marquer profondément les mentalités et la vie des membres de ce groupe. Si P. Judson se réfère surtout au nationalisme culturel et linguistique et se concentre en grande partie sur les Schutzvereine ${ }^{(45)}$, le nationalisme économique est étudié ici, de façon novatrice, dans les associations d'artisans (Gewerbevereine) et de compagnons (Gesellenvereine) saxons et roumains de Kronstadt et Hermannstadt. Ce milieu associatif est d'autant plus intéressant qu'il n'a fait l'objet d'aucune recherche jusqu'à ce jour et permet un regard nouveau sur la coexistence interethnique: d'abord parce que ces associations ne regroupaient pas seulement des artisans, compagnons ou apprentis, mais qu'elles étaient composées de membres appartenant à des couches sociales très diverses: «[...] kein von Handwerkern für Handwerker gebildeter Verein, sondern ein Verein, den Freunde und Gönner des Handwerks zur Förderung desselben errichteten, und zur Mitarbeit in demselben selbstverständlich in

43 Voir Pieter M. Judson, Habsburg. Geschichte eines Imperiums 1740-1918, Munich, C.H. Beck, 2017; voir aussi du même auteur, Guardians of the Nation. Activists on the language frontiers of imperial Austria, Cambridge/London, Harvard Univ. Press, 2006; Rogers BrUBAKer, Ethnizität ohne Gruppen, Hambourg, Hamburger Edition, 2007.

44 R. BRUBAKER, ibid.: «ethnopolitiche Unternehmer».

45 Voir Pieter M. Judson, «Die Schutzvereine und das Grenzland: Strategien zur Verwirklichung von imagined borderlands", in: Peter Haslinger (dir.), Schutzvereine in Ostmitteleuropa. Vereinswesen, Sprachenkonflikte und Dynamiken nationaler Mobilisierung 1860-1939, Marbourg, Verl. HerderInst., 2009. 
erster Linie die Handwerker selbst heranzogen ${ }^{(46)}$. Ces associations avaient d'ailleurs été fondées par des acteurs locaux, représentants d'élites politiques, économiques et religieuses urbaines et régionales. Ensuite, le milieu associatif en question permet une approche du point de vue de l'histoire économique, un champ encore trop peu défriché de la recherche sur la Transylvanie, qui permet de jeter une lumière différente sur les contacts entre Saxons et Roumains («Neben-, Mit- oder Gegeneinander?») ${ }^{(47)}$. Enfin, ces associations de compagnons et d'artisans avaient un dénominateur commun: elles se voulaient lieu de convivialité et d'éducation "nationale» ("edle und bildende Geselligkeit») ${ }^{(48)}$. Elles étaient donc à la fois un lieu important de socialisation (Vergesellschaftung) mais aussi de communautarisation (Vergemeinschaftung). Ces associations avaient été fondées d'après le principe ethno-national et excluaient généralement les membres d'autres groupes. Cependant, cette pratique de la séparation ethnique avait ses limites: ce milieu associatif pouvait être aussi, même épisodiquement, un «lieu» de rencontre (Begegnungsort) entre Saxons et de Roumains.

Même si le tissu associatif saxon et roumain dont il est ici question regroupait des affiliés venant de milieux sociaux très différents, il n'en reste pas moins que les structures restaient très hiérarchiques et l'organisation, patriarcale. Les membres issus de couches sociales défavorisées étaient certes engagés dans les activités de leurs associations, mais seul le comité de direction (Vereinsausschuß), constitué de représentants de l'élite locale, économique, politique et (ou) religieuse (entrepreneurs, fabricants, banquiers, fonctionnaires, prêtres, etc.), prenait les décisions et gérait l'organisation. C'est la raison pour laquelle il est important de compléter les informations des associations par les statistiques de l'industrie et de l'artisanat afin d'étudier l'état des relations roumano-saxonnes dans les classes inférieures de la société urbaine ${ }^{(49)}$. Les statistiques donnent à voir, en outre, la répartition des maîtres-artisans, compagnons ou ouvriers et apprentis d'après l'appartenance ethno-culturelle et permettent de saisir les enjeux du nationalisme dans les deux villes de Transylvanie.

Nationalisme économique et relations entre Roumains et Saxons entre 1868 et 1914, d'une part au sein des associations d'artisans, entre les élites urbaines (von oben), et d'autre part dans les ateliers et usines de Kronstadt et Hermannstadt (von unten), sont modifiés par l'intervention croissante du groupe magyar. Le rôle des Magyars, désormais nation d'État, est déterminant depuis le Compromis austro-hongrois de 1867: d'une part au niveau national ou étatique, à travers la législation visant l'assimilation forcée des populations non-magyares (ou la politique de magyarisation) ainsi que celle portant sur la politique économique et ses conséquences pour les villes transylvaines.

46 Paul Spandowski, Die polnischen Gewerbevereine. Im Rahmen der Entwicklung eines polnischen gewerblichen Mittelstandes, Posen, St. Adalbert-Druckerei und Buchhandlung, 1909, p. 33: «non pas une association d'artisans pour les artisans, mais une association fondée par les amis et protecteurs de l'artisanat afin de le promouvoir, tout en impliquant bien évidemment les premiers concernés, les artisans.»

47 «Coexistence, cohabitation ou confrontation?».

48 «Stiftungsfest des allgemeinen Gesellenvereins», Siebenbürgisch-Deutsches Tageblatt, $\mathrm{n}^{\circ} 14$ (02.09.1868): "une convivialité noble et instructive».

49 «Wie stand es um das Verhältnis "unten” bzw. in den unteren Schichten?», S. DaNneBerG, Wirtschaftsnationalismus lokal (note 1). 
D’autre part, les Magyars interfèrent constamment au niveau local comme concitoyens des Roumains et des Saxons, dans la relation roumano-saxonne. La comparaison micro-historique entre Hermannstadt et Kronstadt démontre que plus la politique de Budapest s'immisçait dans le quotidien des citoyens du royaume et plus la proportion des Magyars dans la population totale des villes transylvaines était élevée, plus les relations des Saxons et des Roumains étaient tendues et plus le nationalisme économique était agressif.

En effet, il existait à Kronstadt et Hermannstadt une bourgeoisie (aussi bien économique qu'intellectuelle) qui était quantitativement très bien dotée, du moins en comparaison avec les chiffres donnés pour les grandes villes européennes ${ }^{(50)}$, mais cette bourgeoisie était presque exclusivement saxonne. Cette domination allemande était propre à de nombreuses villes de l'Europe de l'Est et centrale, même lorsque les Allemands étaient en perte de vitesse démographique. C'est l'exclusivisme ethno-culturel de cette bourgeoisie, ainsi que l'inégale répartition des revenus et des biens, qui furent à l'origine des frustrations sociales et catalysèrent le nationalisme économique des groupes magyar et roumain. Dans les deux villes transylvaines et pendant toute la période du Compromis, l'économie, la gouvernance et la politique locales restèrent aux mains des Saxons, même si les Magyars parvinrent à s'imposer numériquement à Kronstadt. Les perdants de cette répartition furent les Roumains.

Les relations entre les différents groupes de population, dans une Transylvanie culturellement, linguistiquement et confessionnellement hétérogène, constituent pour la longue période du dualisme austro-hongrois (1867-1918) une lacune manifeste de la recherche ${ }^{(51)}$, bien que cette période soit extrêmement intéressante pour l'étude de cette question. La recherche sur la Transylvanie a longtemps été dominée par une historiographie régionale où dominait le désintérêt pour les relations entre les groupes, de sorte que prévalait l'impression d'une Transylvanie roumaine, une Transylvanie hongroise et une Transylvanie saxonne coexistant sans lien entre elles. Cette tradition historiographique avait pris son essor dans la seconde moitié du XIX siècle, lorsque les Transylvains s'étaient mis à écrire «leur» histoire nationale pour leur propre groupe ethno-culturel, comme le souligne l'historien allemand (d'origine saxonne) Harald

50 Voir pour les chiffres: Jürgen KockA, «Das europäische Muster und der deutsche Fall», in: J. KockA (dir.), Bürgertum im 19. Jahrhundert. Deutschland im europäischen Vergleich. Eine Auswahl, t. 1 : Einheit und Vielfalt Europas, Göttingen, Vandenhoeck \& Ruprecht, 1995, p. 10.

51 Dans la Roumanie des années 1960-1980, où, sous Ceausescu, les minorités nationales furent réduites au statut de «nationalités cohabitantes au sein de l'État socialiste unitaire», de nombreux articles portant sur les relations interethniques dans la région transylvaine ont certes été publiés, mais marqués par le sceau de l'idéologie communiste. Une série d'articles portant sur le thème de la "coexistence nationale» est par exemple parue dans la revue Forschungen zur Volks- und Landeskunde, ayant pour but la mise en scène de la lutte «fraternelle» commune contre le «féodalisme» et le «capitalisme». Des ouvrages portant sur la coexistence interethnique et scientifiquement neutres ont été publiés depuis, mais la période du Compromis est de manière générale peu travaillée. L'ouvrage collectif de Sorin Mitu et Florin GogÂltan (Cluj-Napoca, Roumanie) paru en 1996, Interethnische- und Zivilisationsbeziehungen im siebenbürgischen Raum: historische Studien, et dont les contributions pluridisciplinaires vont de l'âge du bronze jusqu'à nos jours, mérite d'être cité dans cette optique. Malgré le titre prometteur de l'ouvrage, seules deux contributions sont consacrées à l'époque austro-hongroise: «Der Bau von Eisenbahnen als eine der Vorbedingungen der Modernisierung in Siebenbürgen» (JANOs) et «Das ungarische Millennium bei den Rumänen» (WEBER). 
Roth: "L'histoire fut rédigée explicitement pour sa propre nation, de sorte que l'existence des "autres" et la cohabitation avec eux ont été très souvent reléguées au second plan» ${ }^{(52)}$. Il s'agit désormais d'écrire l'histoire autrement, en l'envisageant d'un point de vue extérieur - franco-allemand en l'occurrence.

\section{Résumé}

L'article analyse la pratique du nationalisme économique des Roumains et des Saxons dans deux villes de Transylvanie pendant la période du Compromis austro-hongrois (1868-1914), à Hermannstadt/Sibiu et Kronstadt/Braşov, en s'appuyant particulièrement sur la thématique de l'organisation d'expositions artisanales locales. Comme de nombreux autres groupes de population non dominants de la monarchie des Habsbourg, Saxons et Roumains se sont organisés de manière autonome sur le plan économique, au sein d'associations d'artisans, de coopératives et de leurs propres banques; toutes ces structures étant fondées sur le principe ethno-national. L'article porte aussi sur la nature de la coexistence roumano-saxonne qui est en relation étroite avec le rapport de force existant entre ces "nationalités» du royaume de Hongrie et la "Titularnation" des Magyars. Il s'agit au travers de cette micro-histoire locale de contribuer à l'étude du nationalisme économique en Europe centrale, et de mieux comprendre les relations entre les différents groupes de population dans une Transylvanie ethniquement, linguistiquement et confessionnellement hétérogène. L'une des questions centrales de l'argumentation était la suivante: y avait-il des situations et des domaines dans lesquels l'intérêt général économique ou les moments de convivialité pouvaient surmonter le clivage ethnique et créer des liens? L'article traite surtout de la pertinence de la comparaison entre les deux centres urbains Hermannstadt et Kronstadt, de la méthode novatrice de l'analyse d'associations d'artisans comme «lieu» d'expression du nationalisme économique et enfin du rôle moteur des Magyars dans la relation roumano-saxonne («Neben-, Mit- oder Gegeneinander?»).

\section{Zusammenfassung}

Die Studie untersucht, wie Siebenbürger Sachsen und Siebenbürger Rumänen in der Ausgleichsepoche der Habsburgermonarchie (1868-1914) auflokaler Ebene und hier am Beispiel der Organisation von Gewerbeausstellungen, wirtschaftsnationalistisch agierten. Genauso wie andere politisch nichtdominante Bevölkerungsgruppen innerhalb des Habsburgerreichs haben sich Sachsen und Rumänen nach nationalen Kriterien wirtschaftlich „selbst organisiert“: innerhalb von Gewerbevereinen, Genossenschaften und eigenen Banken. Gefragt wird nach der Gestaltung des Verhältnisses zwischen Sachsen und Rumänen, das stets in engem Zusammenhang mit dem Verhältnis zwischen diesen „Nationalitäten“ und der „Titularnation“ der Magyaren in den siebenbürgischen Zentren Hermannstadt und Kronstadt zu sehen ist. Hier wird sowohl ein Beitrag zum ostmitteleuropäischen Wirtschaftsnationalismus als auch zum Verständnis des Verhältnisses

52 Harald Roth, Kleine Geschichte Siebenbürgens, Cologne, Böhlau, 2003 (2 éd.), p. 156: "Geschichte wurde nun ausdrücklich für das eigene Volk geschrieben, so daß die Existenz andersnationaler Nachbarn und das Zusammenleben mit diesen nicht selten ganz in den Hintergrund rückten.» 
zwischen den verschiedenen Bevölkerungsgruppen im ethnisch, sprachlich und religiös heterogenen Siebenbürgen angestrebt. Leitfragen der Argumentation waren unter anderem: Gab es Bereiche und Situationen, in denen das wirtschaftliche Gemeinwohl oder die Geselligkeit die Bevölkerungsgruppen verband, wo sonst die Ethnizität trennte? Welche Anknüpfungspunkte, Gemeinsamkeiten oder Unterschiede gab es hinsichtlich wirtschaftsnationalistisch geprägter Werthaltungen in Ostmitteleuropa? Hier werden insbesondere die Relevanz des Vergleichs zwischen den zwei Zentren Hermannstadt und Kronstadt, die wegweisende Analyse von Gewerbeorganisationen als Vermittler des „innerstaatlichen“ Wirtschaftsnationalismus sowie die Magyaren als Tertium Comparationis (Rumänen und Sachsen: Neben-, Mit-oder Gegeneinander?) vorgestellt.

\begin{abstract}
The study analyses how, on the local level, Transylvanian Saxons and Romanians acted economic-nationalistically in the Habsburg Monarchy during the Austro-Hungarian Compromise period (1868-1914), introduced here through the analysis of trade/industrial exhibitions. The Saxons and Romanians were just like other political non-dominant ethnic groups inside the Habsburg Empire economically self-organized along national lines: within trade-associations, cooperatives and independent banks. It is also about the arrangement of the relationship between Transylvanian Saxons and Romanians, always closely linked to the relationship between these "nationalities" and the Magyar "titular nation" in the Transylvanian centers Sibiu/Hermannstadt and Brasov/Kronstadt. The micro-historical study aims at a contribution to both East-Central European economic nationalism and to the understanding of the coexistence between the different population groups in ethnically, linguistically and religiously heterogeneous Transylvania. One of the central questions of the argumentation was: Were there any fields or situations in which the economic welfare or sociability united population groups that were otherwise separated by ethnicity? The article introduces the pertinence of the comparison between Sibiu and Brasov, the term of ,intrastate” economic nationalism here analyzed in trade-associations - a path-breaking method - and the role of the Magyars as Tertium Comparationis and "driving-force” behind the relationship between Saxons and Romanians (coexistence, cooperation or conflict?).
\end{abstract}

